\title{
Robot-assisted Laparoscopic Nephroureterectomy for a Nephrogenic Adenoma of Ureter
}

\author{
Keiran David Clement, Catherine Thompson and Omar M. Aboumarzouk \\ Department of Urology, Queen Elizabeth University Hospital, Glasgow, Scotland, United Kingdom
}

\begin{abstract}
Nephrogenic adenoma is a rare and benign tumour of the urinary tract thought to be caused by metaplastic change of native urothelial tissue. The majority of cases arise in the bladder, with very few cases affecting the ureter reported in the literature. Herein, we describe the presentation, diagnostic challenges, and the eventual management of a nephrogenic adenoma of the ureter by robot-assisted laparoscopic nephroureterectomy. Urologists and pathologists should be aware of the potential diagnostic and management pitfalls associated with this rare tumour, as well as the sparsity of evidence with respect to follow-up.
\end{abstract}

Key Words: Robotic surgery, Nephroureterectomy, Nephrogenic adenoma.

How to cite this article: Clement KD, Thompson $C$ and Aboumarzouk OM. Robot-assisted laparoscopic nephroureterectomy for a nephrogenic adenoma of ureter. J Coll Physicians Surg Pak 2019; 29 (Supplement 2):S151-S153.

\section{INTRODUCTION}

Nephrogenic adenoma (NA) is a rare benign tumour of the urinary system, so named due to its tubular nature when reviewed microscopically. Fewer than 30 cases affecting the ureter have been reported in the literature. 1 Herein, we describe the presentation, assessment and management of NA of the ureter through robot-assisted laparoscopic nephroureterectomy (RALNU).

\section{CASE REPORT}

A 52-year female was admitted with urinary sepsis. A CT scan demonstrated right hydronephrosis, proximal hydroureter and the suspicion of a five centimetre malignant stricture of distal right ureter (Figure 1). The patient reported right-sided abdominal pain for several years, which had not previously been investigated. Abdominal examination was normal. The patient had a background of insulin-controlled type 2 diabetes, pulmonary tuberculosis, and depression.

On discharge, she was referred for an outpatient rigid ureteroscopy and MAG3 renogram, but failed to attend. Following this, she was readmitted with suprapubic pain radiating to her right iliac fossa and back, as well as persistent visible haematuria. She was treated with oral antibiotics for a presumed urinary tract infection and was re-referred for an outpatient diagnostic ureteroscopy.

Correspondence to: Dr. Keiran David Clement, Department of Urology, Queen Elizabeth University Hospital, Glasgow,

Scotland, UK

E-mail: keiranclement@nhs.net

Received: May 18, 2019; Revised: July 17, 2019;

Accepted: July 27, 2019
Five months after her initial CT scan, she underwent rigid cystoscopy and right ureteroscopy. On fluoroscopy, it was evident that there was a filling defect within the lower third of the ureter (Figure 2), and ureteroscopy was suspicious for a malignant process, which was subsequently biopsied. Pathological assessment demonstrated fragments of urothelium with chronic inflammation, congestion and vascular thrombosis. There was formation of papillae with a covering of bland low cuboidal cells with some hobnailing. There was no epithelial thickening or atypia in the sample, and no evidence of infiltrative growth. The findings were consistent with a benign NA and a papillary pattern.

The patient was concerned about the potential of malignant transformation. She was counselled extensively regarding the high likelihood that the lesion was benign in nature. Despite this, the patient was keen to have the lesion surgically removed due to ongoing concern for its malignant potential.

She underwent a right RALNU seven months following her initial CT scan. Using the Da Vinci Xi System, the patient was placed in a modified flank position using a special cushioned brace with a groove for the shoulder and the table-sided arm is placed outwards. A 12-mm assistant port is placed in the midline, $5 \mathrm{~cm}$ cranial to the umbilicus. Pneumoperitoneum was then established. Four robotic ports were then placed under direct videoscopic vision. Port 1 was placed $2 \mathrm{~cm}$ below the ribs at the edge of the rectus sheath. Port 4 was placed midway between the symphysis pubis and the umbilicus in the midline. The remaining ports were placed in a linear alignment, with $6-8 \mathrm{~cm}$ between each of the 4 ports. The targeting was done midway between the lliac vessels and the lower pole of the kidney. Port 1 held the 
monopolar scissors, Port 2 the camera, Port 3 bipolar fenestrated graspers, and Port 4 the Prograsp forceps. A needle holder was placed in Port 3 and the graspers in Port 1 for the bladder closure.

The bowel was mobilised, the ureter was identified early and clipped. Then dissection of the ureter was done down to the bladder. During the operation, she was found to have significant fatty, inflammatory adhesions throughout her urinary tract. Her lower ureter was fused to pelvic wall with extensive neovascularisation warranting extension of one of her robotic port sites to allow direct visualisation and safe dissection down to the ureteric orifice. A bladder cuff was taken and the bladder sutured water-tight with a double layered closure using V-Lock suture. A leak test was carried out. The nephrectomy was then carried out.

Postoperatively, she remained in hospital for 13 days due to analgesic issues and a persistently low haemoglobin. A follow-up CT scan showed no evidence to suggest active bleeding. She was reviewed as an outpatient three months postoperatively; and found to be pain-free and have normal renal function.

Gross pathological assessment of the nephroureterectomy specimen demonstrated a kidney with a shrunken, fibrotic parenchyma and a dilated collecting system. There were no renal lesions evident. The distal ureter appeared macroscopically normal.

Microscopically, the sections from the proximal and distal ureter demonstrated a proliferation of small tubules lined by a mixture of cuboidal and hobnail epithelium, some of which contained thyroid-like secretions. The majority of the proliferation was within the lamina propria of the ureter, but there was focal involvement of the muscularis propria. There was minimal cytological atypia and no mitotic figures were evident.

Immunostaining was performed and was negative for AMACR and PSA, but positive for PAX8. The proliferation was assessed by Ki-67 staining, which was found to be extremely low. The appearances were again consistent with the initial ureteroscopic biopsies, found to be of a benign NA. The patient provided informed verbal consent for submission of this case report.

\section{DISCUSSION}

$\mathrm{NA}$ is a benign lesion predominantly affecting males with a sex ratio of 2:1.1 They are found to occur at any age but are thought to present typically in the fourth to sixth decade. ${ }^{2}$ It has been found to occur anywhere in the urinary tract with approximately $55 \%$ of the lesions affecting the bladder, while only $4 \%$ affect the ureter. ${ }^{3}$

It is thought to be caused by chronic inflammatory states in the urinary tract such as those associated with trauma, chronic infection, renal transplantation, urinary stones, urinary tract surgery, and extensive instrumentation. ${ }^{1}$ In this case, the most likely cause of chronic inflammation was recurrent upper urinary tract infection, although given the patient's relative propensity to avoid medical assessment, this was only speculation.

Presentation is varied depending on the site of the lesion. Symptomatology can include haematuria, dysuria, nocturia, urinary frequency, renal colic and microscopic hematuria. ${ }^{4}$ In one review of the literature, most patients found to have NA of the bladder initially presented with irritative symptoms of the lower urinary tract, such as urinary frequency and urgency; but rarely hematuria. ${ }^{1}$ The patient in our case had a long, protracted history of vague right-sided abdominal pain, and was found to have an obstructive uropathy only after undergoing a CT urogram (Figure 1) to investigate presumed urinary sepsis. Other literature reports have previously noted the preponderance for NA to be discovered incidentally by other means of imaging endoscopy or pathologically. ${ }^{3}$

Lesions examined microscopically tend to reveal small hollow tubules, which appear similar to mesonephric

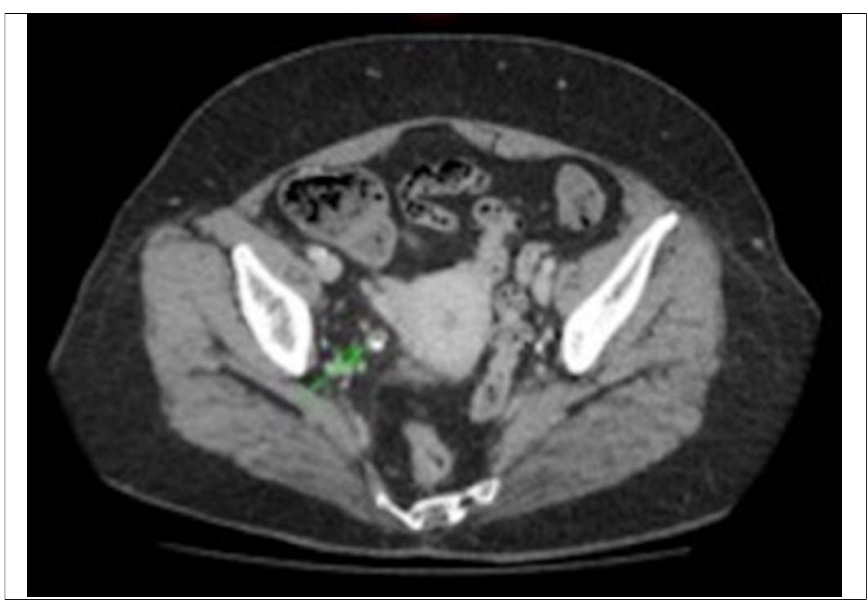

Figure 1: CT urogram demonstrating a right ureteric filling defect.

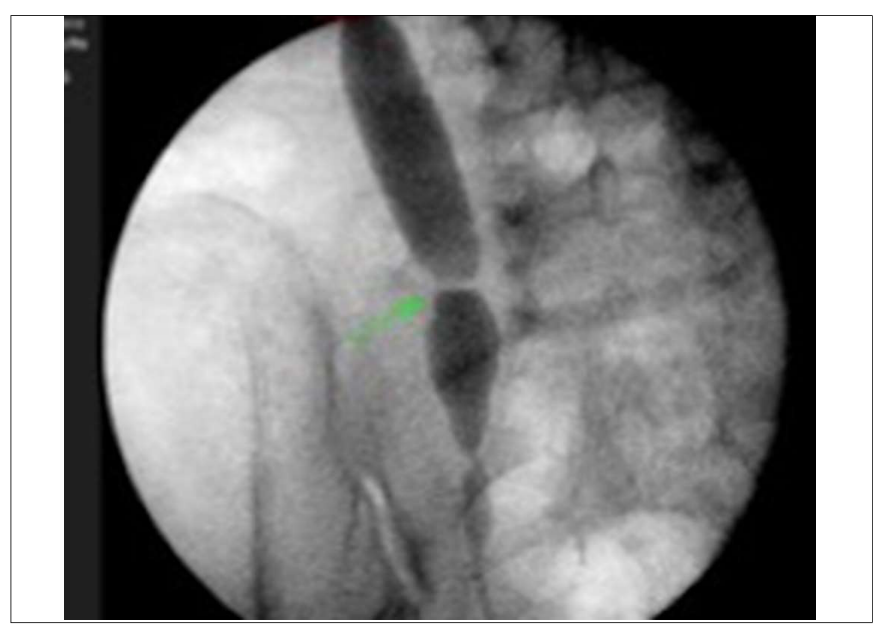

Figure 2: Intraoperative fluoroscopy demonstrating a right ureteric filling defect. 
tubules and are lined by bland cuboidal or hobnail cells. 4 This was apparent in our patient's tumour. There is generally involvement of the lamina propria with sparing of the muscularis propria. There was minimal atypia and minimal mitotic figures in assessment of this patient's tumour, which is characteristic of a non-malignant process. ${ }^{4} \mathrm{NA}$ is thought to have no malignant potential, and there are no cases in the literature to suggest this. However, there is one report of recurrent NA; therefore, repeat cystoscopic examinations for follow-up is suggested. 1

In conclusion, in this case, we describe the presentation, diagnostic challenges and eventual RALNU that was performed. Urologists and pathologists should be aware of the potential diagnostic and management pitfalls associated with this rare disease, as well as the sparsity of evidence with respect to follow-up.

\section{PATIENT'S CONSENT:}

The patient provided informed verbal consent for publication of this case report

\section{CONFLICT OF INTEREST:}

Authors declared no conflict of interest.

\section{AUTHORS' CONTRIBUTION:}

OA: Proposed and supervised the publication of this case report, approved the manuscript prior to publication.

KDC, CT: Undertook data collection, literature review and drafting of the text, approved the manuscript prior to publication.

\section{REFERENCES}

1. Venyo AK. Nephrogenic adenoma of the urinary bladder: A review of the literature. Int Sch Res Notices 2015; 2015: 704982.

2. Jackman SV, Moore RG, Nelson JB. Nephrogenic adenoma of the ureter: Endoscopic diagnosis and management. Urology 1998; 52:316-317.

3. Oliva E, Young $\mathrm{RH}$. Nephrogenic adenoma of the urinary tract: A review of the microscopic appearance of 80 cases with emphasis on unusual features. Mod Pathol 1995; 8:722-30.

4. Doluoglu OG, Acarer EY, Yavuz A, Hoscan MB, Gonulalan U. Nephrogenic adenoma of the ureter. Rare Tumours 2012; 4:e28. 